\title{
The current status and future perspectives of clinical trial groups in Japan
}

\author{
Hirofumi Mukai
}

Published online: 24 August 2013

(C) The Japanese Breast Cancer Society 2013

Our country's environment surrounding clinical trials in the breast cancer field is characterized by the following; there are a lot of small clinical trial groups, most of which have no data center of their own; trial design and statistical analysis is shared by statisticians in a limited number of these groups, in the majority of which trial-participating physicians themselves are engaged in statistical analyses.

A large number of trial groups perform only a phase II study because they cannot practically implement a phase III study which has to recruit a number of patients becoming necessarily large in scale, and because a phase I study requires specialists with knowledge as well as skills of such a study. In Japan, there is almost no culture where patient accrual is promoted by a key physician in a local community who is asked about it. Unfortunately, the state of affairs now is such that any trial is not always satisfactorily progressing in any group.

Naturally, trial implementation necessitates operational expenses, which are likely to be covered by donations from pharmaceutical companies in the situation of our country where a sufficient amount of public research grants are not provided for clinical trials. Financial support offered by pharmaceutical companies has also a strong implicit intention to promote the sale of their products. While leading physicians work without recompense on a clinical trial, pharmaceutical companies sponsoring it advertise massively their products during the trial.

Since these kinds of trials are carried out in every part of our country, they are extremely inefficient in the sense that trials whose concepts are much like each other are in

H. Mukai $(\bowtie)$

Division of Oncology/Hematology, National Cancer Center Hospital East, 6-5-1 Kashiwanoha, Kashiwa-shi, Chiba, Japan e-mail: hrmukai@east.ncc.go.jp progress at the same time in lots of areas. This situation is not acceptable in terms of the clinical trials' original purpose of giving back reliable results quickly to patients through accomplishing clinical trials speedily in a proper methodical manner. This situation is also problematic from the aspect of the ethics of the clinical trial. However, it is expected to undergo a complete change in the near future due to "Transparency guidelines concerning the relationship between corporate activities and medical institutions" issued by the Japan Pharmaceutical Manufacturers.

In other words, it is expected that many of small clinical trial groups in Japan will be dumped into the dustbin and that only groups that are able to accomplish research projects with definite philosophy as well as purpose will survive.

It is an obvious fact that the performance of cancer treatment has been improved partially because of the introduction of many excellent drugs to medical practice. It is drug development followed by registration trials that plays a role for new drug introduction. However, in consideration of the reality that medical treatment is usually combined with multidrug therapy, surgical therapy or radiation therapy, newly introduced drugs cannot be usually used as they are in the clinical practice of cancer therapy.

New drugs are absolutely required to undergo a process in which they are explored for combination with other drugs and optimal use in combination with other therapies to establish better their positions. Investigator initiated clinical trials must assume a large role in the application of newly marketed drugs to the standard therapy.

At present, the reliability of investigator initiated clinical trials as a whole has been damaged in Japan because of suspected manipulation of clinical study data on Valsartan manufactured by Novartis Pharma. Although investigator 
initiated clinical trials shall not lose their necessity as well as great significance, investigators need to rebuild trust from the society by more strictly securing transparency and achieving accountability from now on.

In our country, investigator initiated clinical trials have not been controlled by Good Clinical Practice (GCP) until today. The suspected data manipulation as well as the worldwide trend makes it possible that such trials, like registration trials for drug development, will be regulated by GCP. Only clinical trial groups that can cope with that situation will globally play an active role in the future. 